\title{
Expression Changes in Lactate and Glucose Metabolism and Associated Transporters in Basal Ganglia following Hypoxic-Ischemic Reperfusion Injury in Piglets
}

\author{
(D) Y. Zheng and (D) X.-M. Wang
}

\begin{abstract}
BACKGROUND AND PURPOSE: The neonatal brain has active energy metabolism, and glucose oxidation is the major energy source of brain tissue. Lactate is produced by astrocytes and released to neurons. In the central nervous system, lactate is transported between neurons and astrocytes via the astrocyte-neuron lactate shuttle. The aim of this study was to investigate the regulatory mechanisms of energy metabolism in neurons and astrocytes in the basal ganglia of a neonatal hypoxic-ischemic brain injury piglet model.

MATERIALS AND METHODS: A total of 35 healthy piglets (3-5 days of age; $1.0-1.5 \mathrm{~kg}$ ) were assigned to a control group ( $n=5$ ) or a hypoxic-ischemic model group $(n=30)$. The hypoxic-ischemic model group was further divided into 6 groups according to the ${ }^{1} \mathrm{H}-\mathrm{MR}$ spectroscopy and PET/CT scan times after hypoxia-ischemia (0-2, 2-6, 6-12, 12-24, 24-48, and 48-72 hours; $n=5 /$ group). ${ }^{1} \mathrm{H}-\mathrm{MR}$ spectroscopy data were processed with LCModel software. Maximum standard uptake values refer to the maximum standard uptake values for glucose (or FDG). The maximum standard uptake values of the basal ganglia-to-occipital cortex ratio were analyzed. The expression levels of glucose transporters and monocarboxylate transporters were detected by immunohistochemical analysis.

RESULTS: Lactate levels decreased after an initial increase, with the maximal level occurring around 2-6 hours following hypoxia-ischemia. After hypoxia-ischemia, the maximum standard uptake values of the basal ganglia and basal ganglia/occipital cortex initially increased then decreased, with the maximum occurring at approximately 6-12 hours. The lactate and glucose uptake (basal ganglia/occipital cortex maximum standard uptake values) levels were positively correlated. The expression levels of glucose transporter-1 and glucose transporter-3 were positively correlated with the basal ganglia/occipital cortex. The expression levels of monocarboxylic acid transporter-2 and monocarboxylic acid transporter-4 were positively correlated with lactate content.
\end{abstract}

CONCLUSIONS: The results indicate that lactate and glucose transporters have a synergistic effect on the energy metabolism of neurons and astrocytes following hypoxic-ischemic reperfusion brain injury.

ABBREVIATIONS: ANLS = astrocyte-neuron lactate shuttle; $B G=$ basal ganglia; $G L U T=$ glucose transporter; $\mathrm{HI}=$ hypoxic-ischemic; $M C T=$ monocarboxylic acid transporter; $\mathrm{OC}=$ occipital cortex; $\mathrm{SUV}_{\max }=$ maximum standard uptake values

$\mathbf{R}$ ecent studies of the astrocyte-neuron lactate shuttle (ANLS) suggest that lactate is generated and released by astrocytes in the process of glucose metabolism and is further taken up and metabolized by neurons as an oxidizing substrate in energy me-

Received May 9, 2017; accepted after revision October 30

From the Department of Radiology, Shengjing Hospital of China Medical University, Shenyang, PR China.

This study was supported by the National Natural Science Foundation of China (No. 30570541, 30770632, 81271631) and the Outstanding Scientific Fund of Shengjing Hospital (No. 201402).

Please address correspondence to Xiaoming Wang, MD, Department of Radiology, Shengjing Hospital of China Medical University, No. 36, Sanhao St, Heping District, Shenyang 110004, PR China; e-mail address: wangxm024@163.com; @little_yangyang

- Indicates open access to non-subscribers at www.ajnr.org

http://dx.doi.org/10.3174/ajnr.A5505 tabolism in the brain. ${ }^{1,2}$ Lactate metabolism provides adenosine triphosphate to neurons under hypoxic conditions. ${ }^{3}$ The ANLS requires monocarboxylic acid transporters on the plasma membranes of both neurons and astrocytes for transfer of lactate, pyruvate, and other monocarboxylic acids, as well as acetoacetic acid, $\beta$-hydroxybutyric acid, and so forth. ${ }^{4}$ Astrocytes and neurons express different monocarboxylate transporter (MCT) subtypes, because astrocytes primarily express MCT-4 and neurons primarily express MCT-2. ${ }^{5}$ Lactate and its transporters play important roles in cell metabolism under hypoxic-ischemic conditions. ${ }^{2}$ The transport and uptake of lactate between astrocytes and neurons are mediated by MCTs. Briefly, lactate is transported out of astrocytes by membrane-bound MCT- 4 and gathers in the extracellular compartment. Neurons then take up lactate through membrane-bound MCT-2, which is then converted to acetone by 


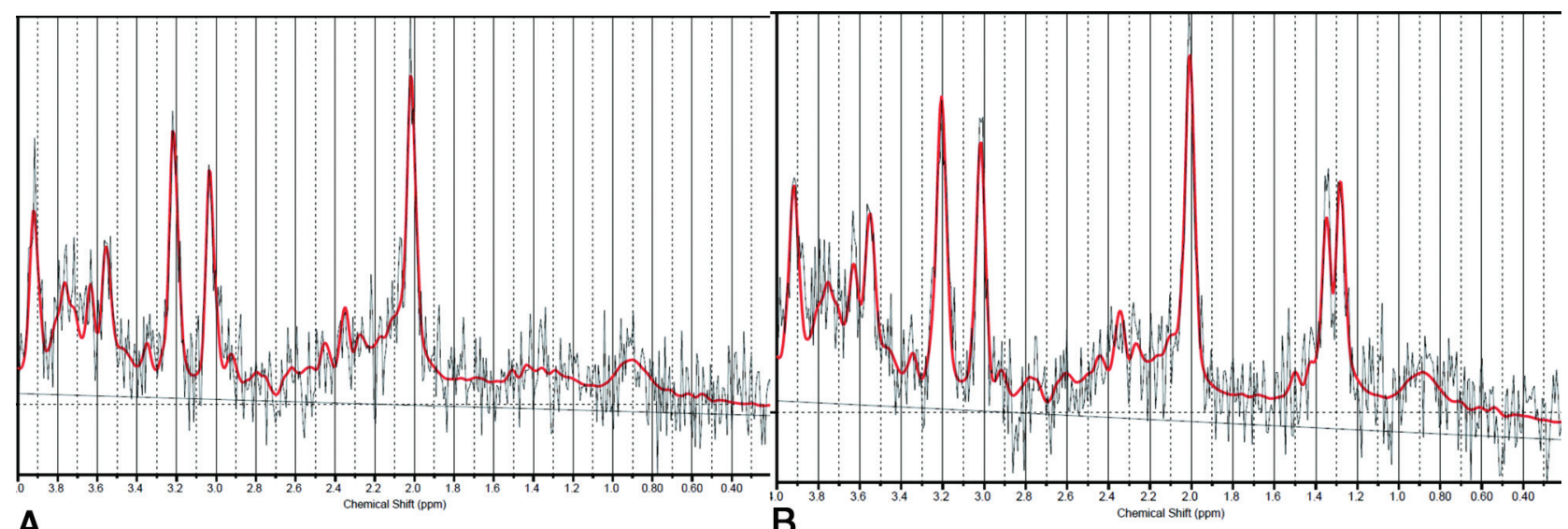

A

B

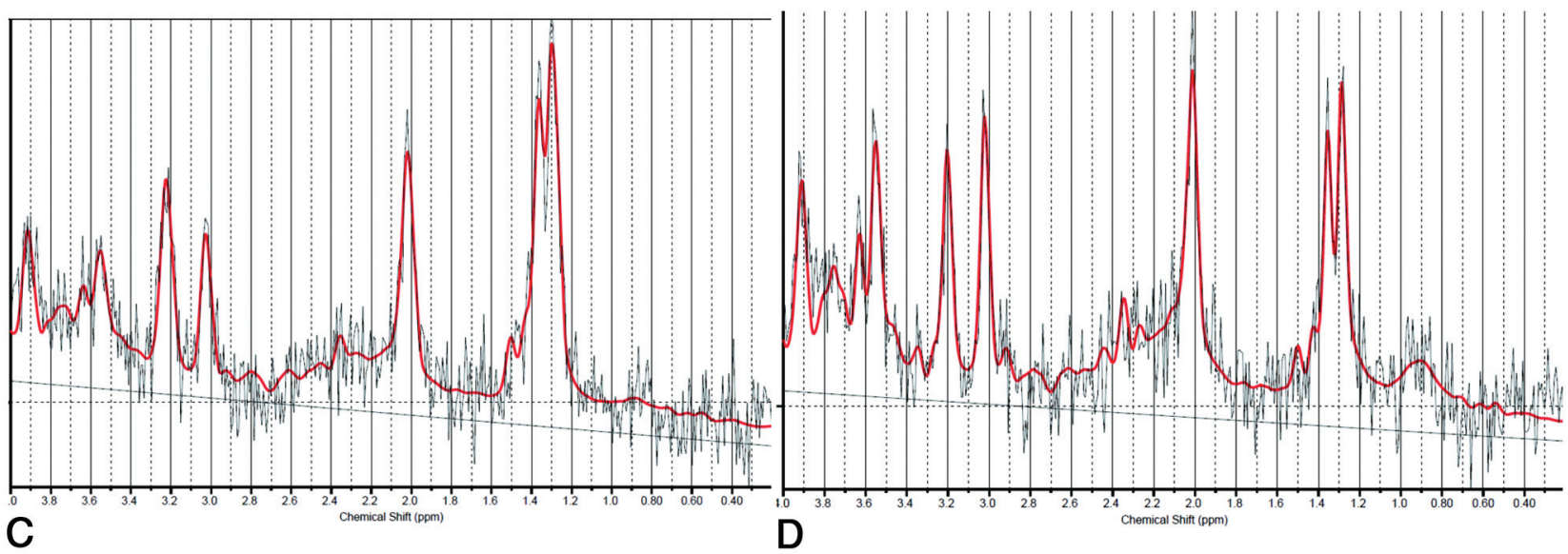

C

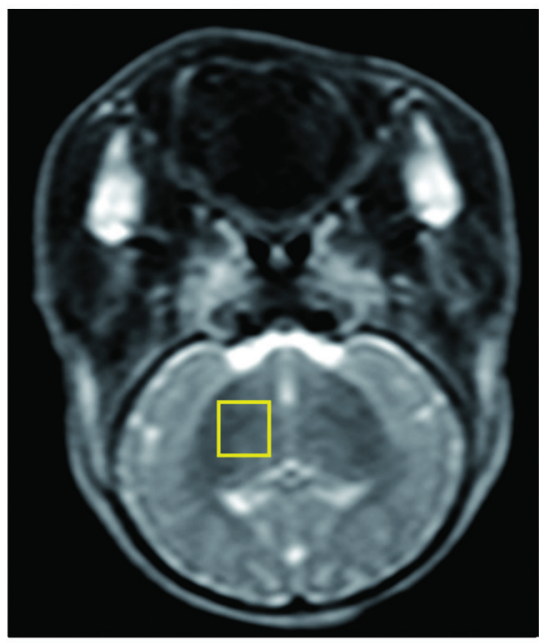

$\mathrm{E}$

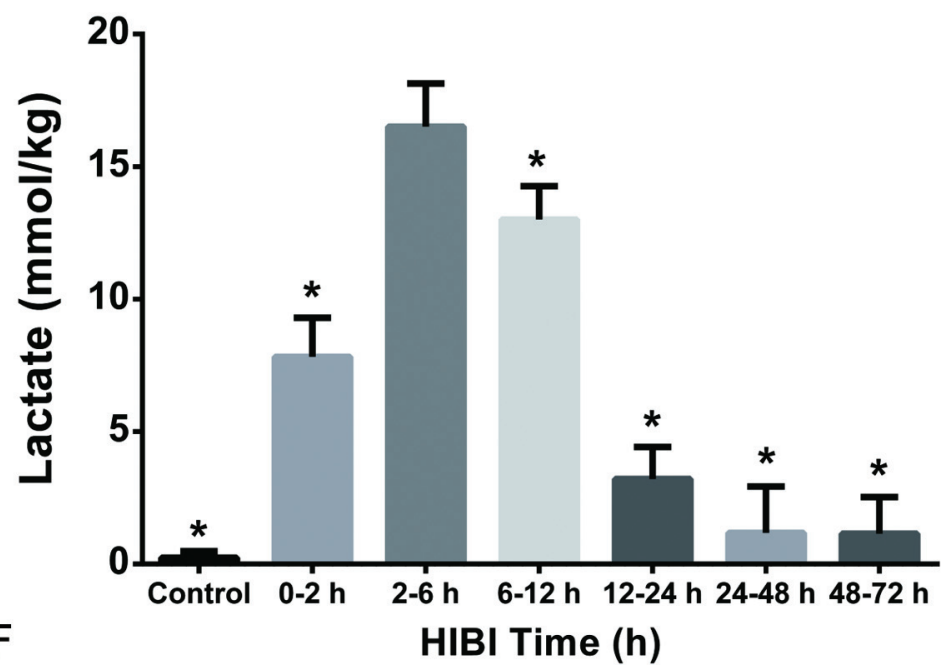

FIG 1. Changes in ${ }^{1} \mathrm{H}-\mathrm{MR}$ spectroscopy spectra and lactate content in the BG within 72 hours after HI. ${ }^{1} \mathrm{H}$-MR spectroscopy spectra of the right BG of the control and HI model groups at $0-2,2-6$, and $6-12$ hours are shown as $A-D$ (lactate peak is located at 1.33 ppm). The lactate peak in the control group is low and flat $(A)$ and peaked at 2-6 hours after $\mathrm{HI}(C)$. ROI selection (E): right BG (yellow solid red). The change in lactate content after $\mathrm{HI}$ is shown in $F$ (we calibrated the results, assuming $80 \%$ brain-water content, $100 \%$ visibility of water signal, and 55.5 mol/L). The asterisk represents a statistical difference compared with the 2- to 6-hour time point. HIBI indicates hypoxic-ischemic brain injury.

lactate dehydrogenase, and acetone is then metabolized aerobically via the citric acid cycle to produce energy. ${ }^{5-9}$

Moreover, lactate is closely associated with glucose metabolism, but a correlation with dynamic metabolic changes remains unclear. In vivo glucose metabolism in the brain is evaluated by $\left[{ }^{18} \mathrm{~F}\right]$ fluorodeoxyglucose imaging. In this study, ${ }^{1} \mathrm{H}-\mathrm{MR}$ spec- troscopy in combination with $\left[{ }^{18} \mathrm{~F}\right]$ FDG imaging was used to observe changes in lactate and glucose levels at different time points after hypoxic-ischemic (HI) in a piglet model. Histologic analysis demonstrated the existence of a mutual regulatory mechanism of energy metabolism in astrocytes and neurons after HI. 
Levels of lactate, SUV max $_{\text {MCTs, and GLUTs in the control and HI model groups }}{ }^{\mathrm{a}}$

\begin{tabular}{|c|c|c|c|c|c|c|c|}
\hline \multirow[b]{2}{*}{ Parameter } & \multirow{2}{*}{$\begin{array}{l}\text { Control } \\
(n=5)\end{array}$} & \multicolumn{6}{|c|}{ HI Model Group ( $n=5 /$ Group) } \\
\hline & & $0-2 \mathrm{hr}$ & $2-6 \mathrm{hr}$ & $6-12 \mathrm{hr}$ & $12-24 \mathrm{hr}$ & $24-48 \mathrm{hr}$ & $48-72 \mathrm{hr}$ \\
\hline Lactate & $0.24 \pm 0.25$ & $7.82 \pm 1.48^{b}$ & $16.51 \pm 1.62^{b}$ & $13.01 \pm 1.25^{\mathrm{b}}$ & $3.21 \pm 1.21^{\mathrm{b}}$ & $1.18 \pm 1.74$ & $1.23 \pm 1.40$ \\
\hline $\mathrm{BG} / \mathrm{OC}$ & $0.99 \pm 0.01$ & $1.08 \pm 0.04^{b}$ & $1.17 \pm 0.06^{b}$ & $1.23 \pm 0.02^{b}$ & $1.16 \pm 0.03^{b}$ & $1.04 \pm 0.04^{b}$ & $0.96 \pm 0.01$ \\
\hline MCT-2 & $0.19 \pm 0.00$ & $0.21 \pm 0.01^{\mathrm{b}}$ & $0.22 \pm 0.01^{\mathrm{b}}$ & $0.24 \pm 0.01^{\mathrm{b}}$ & $0.27 \pm 0.01^{\mathrm{b}}$ & $0.21 \pm 0.01^{b}$ & $0.19 \pm 0.00$ \\
\hline MCT-4 & $0.21 \pm 0.01$ & $0.24 \pm 0.01^{\mathrm{b}}$ & $0.25 \pm 0.01^{\mathrm{b}}$ & $0.26 \pm 0.01^{\mathrm{b}}$ & $0.27 \pm 0.01^{\mathrm{b}}$ & $0.25 \pm 0.02^{b}$ & $0.22 \pm 0.02$ \\
\hline GLUT-1 & $0.14 \pm 0.02$ & $0.16 \pm 0.04$ & $0.20 \pm 0.05^{b}$ & $0.25 \pm 0.01^{\mathrm{b}}$ & $0.24 \pm 0.03^{b}$ & $0.18 \pm 0.05$ & $0.15 \pm 0.05$ \\
\hline GLUT-3 & $0.13 \pm 0.00$ & $0.13 \pm 0.01$ & $0.16 \pm 0.01^{b}$ & $0.22 \pm 0.06^{b}$ & $0.18 \pm 0.01^{b}$ & $0.14 \pm 0.01^{b}$ & $0.14 \pm 0.01$ \\
\hline
\end{tabular}

Data are mean \pm SD

${ }^{\mathrm{b}} P<.05$ versus the control group.

\section{MATERIALS AND METHODS Experimental Animals}

A total of 35 healthy Yorkshire or Large White piglets (3-5 days of age; $1.0-1.5 \mathrm{~kg}$ ) were randomly divided into 7 groups of 5 piglets each: a control group and $6 \mathrm{HI}$ model groups. All animal experiments satisfied the standards of the Regulations for the Administration of Affairs Concerning Experimental Animals and Measures for the Administration of Licenses Concerning Experimental Animals.

\section{Establishment of the HI Model}

Control Group. Room temperature was maintained at $28^{\circ} \mathrm{C}-$ $30^{\circ} \mathrm{C}$. The animals were injected intramuscularly with $0.6 \mathrm{~mL} / \mathrm{kg}$ of Su-Mian-Xin (Veterinary Research Center, Jilin University, Changchun, China). ${ }^{10}$ Each animal was mechanically delivered $100 \%$ oxygen with a TKR-200C small animal ventilator (Jiangxi TELI Anesthesia \& Respiration Equipment, Nanchang City, China) using a 2.5-mm-diameter endotracheal tube. The parameters of the ventilator were the following: inspiration-to-expiration ratio, 1:1.5; respiratory rate, 30 breaths/min; pressure, $0.05-0.06 \mathrm{MPa}$. A handheld TUFFSAT Pulse Oximeter (GE Healthcare, Milwaukee, Wisconsin) was used to monitor heart rate and oxygen saturation. After the neck skin was disinfected with iodophor, the jugular vein was catheterized and an operation was performed to free the bilateral common carotid arteries. Then, the incisions were sutured, and the animals were placed in an incubator to maintain body temperature.

Model Groups. During the first stage, animals in the model groups underwent the same procedures as those in the control group. In the second stage, following 30 minutes of stabilization, artery clamps were used to block the bilateral common carotid arteries. Simultaneously, the oxygen concentration administered by mechanical ventilation was decreased to $6 \%$ (mixed in $94 \%$ nitrogen; Dalian Special Gases, Dalian City, China), which was maintained for 40 minutes and then increased to $100 \%$. Both clamps on the bilateral common carotid arteries were then removed, blood flow was recovered, and the incisions were sutured. Oxygen saturation and heart rate were monitored throughout the procedure. Mechanical ventilation was stopped after spontaneous breathing was restored.

There were 7 groups ( $n=5$ piglets each): a control group and 6 model groups. The $6 \mathrm{HI}$ model groups were based on PET/CT and MR spectroscopy scanning times after hypoxia-ischemia (0-2, 2-6, 6-12, 12-24, 24-48, and 48-72 hours). The model group was standardized, in which case, the HI time and surgical procedure of each neonate piglet in the model group were the same. The piglets in the control group underwent the same operation without $\mathrm{HI}$ process.
According to the preoperative, operative, and postoperative monitoring of the hypoxic-ischemic brain injury model, partial pressure of carbon dioxide in arterial blood was maintained in the range of $4.5-6.5 \mathrm{kPa}$, and partial pressure of oxygen in arterial blood, in the range of $8-13 \mathrm{kPa}{ }^{11}$

After completion of PET/CT and MR spectroscopy at the designated time points, the piglets were immediately injected with anesthetics. After the reaction disappeared (ie, loss of pain reflex because of excessive anesthetic), the skull was opened along the sutura craniun, and the spinal cord was cut at the medulla. The whole brain was collected along with the cerebellum after the piglet was decapitated.

\section{${ }^{1}$ H-MR Spectroscopy Scanning and Data Processing}

${ }^{1} \mathrm{H}-\mathrm{MR}$ spectroscopy scans were performed of control group and at $0-2,2-6,6-12,12-24,24-48$, and $48-72$ hours after HI.

MR imaging was performed with an Achieva 3T TX system (Philips Healthcare, Best, the Netherlands) equipped with a pencil beam, second-order shim. Body coil emission and 8-channel sensitivity encoding head coil reception were used. ${ }^{1} \mathrm{H}-\mathrm{MR}$ spectroscopy was implemented with a point-resolved spectroscopy singlevoxel sequence with the following scanning parameters: TR/TE, 2000/37 ms; average number of signals, 64 ; and VOI, $10 \times 10 \times 10$ $\mathrm{mm}$. The right basal ganglia (BG) was chosen as the ROI. Shimming and water suppression were automatically performed by the scanner. ${ }^{1} \mathrm{H}-\mathrm{MR}$ spectroscopy results were analyzed using the LCModel software package (http://www.lcmodel.com/). ${ }^{12}$

\section{PET/CT Studies}

$\mathrm{PET} / \mathrm{CT}$ scans were performed of control group and at $0-2,2-6$, $6-12,12-24,24-48$, and $48-72$ hours after HI.

\section{PET Reagents}

$\left[{ }^{18} \mathrm{~F}\right]$ FDG was synthesized using the MINItrace positron-emitting isotope production system (GE Healthcare) and a TracerLab MX-FDG synthesizer (GE Healthcare). High-performance liquid chromatography used for quality control showed that the radioactive purity of $\left[{ }^{18} \mathrm{~F}\right]$ FDG was $>99 \%$.

\section{Functional Imaging}

$\left[{ }^{18} \mathrm{~F}\right]$ FDG (3.7 MBq/kg; injection dose, 3.7-5.55 MBq) was administered to the experimental animals in the model groups via the jugular vein at different time points after HI. Feeding was stopped 6 hours before administration to maintain low levels of blood glucose. The radioactivity of the syringe before and after injection was measured. PET/CT scanning (Discovery Elite PET/CT system; GE Healthcare) was performed 50 minutes after 

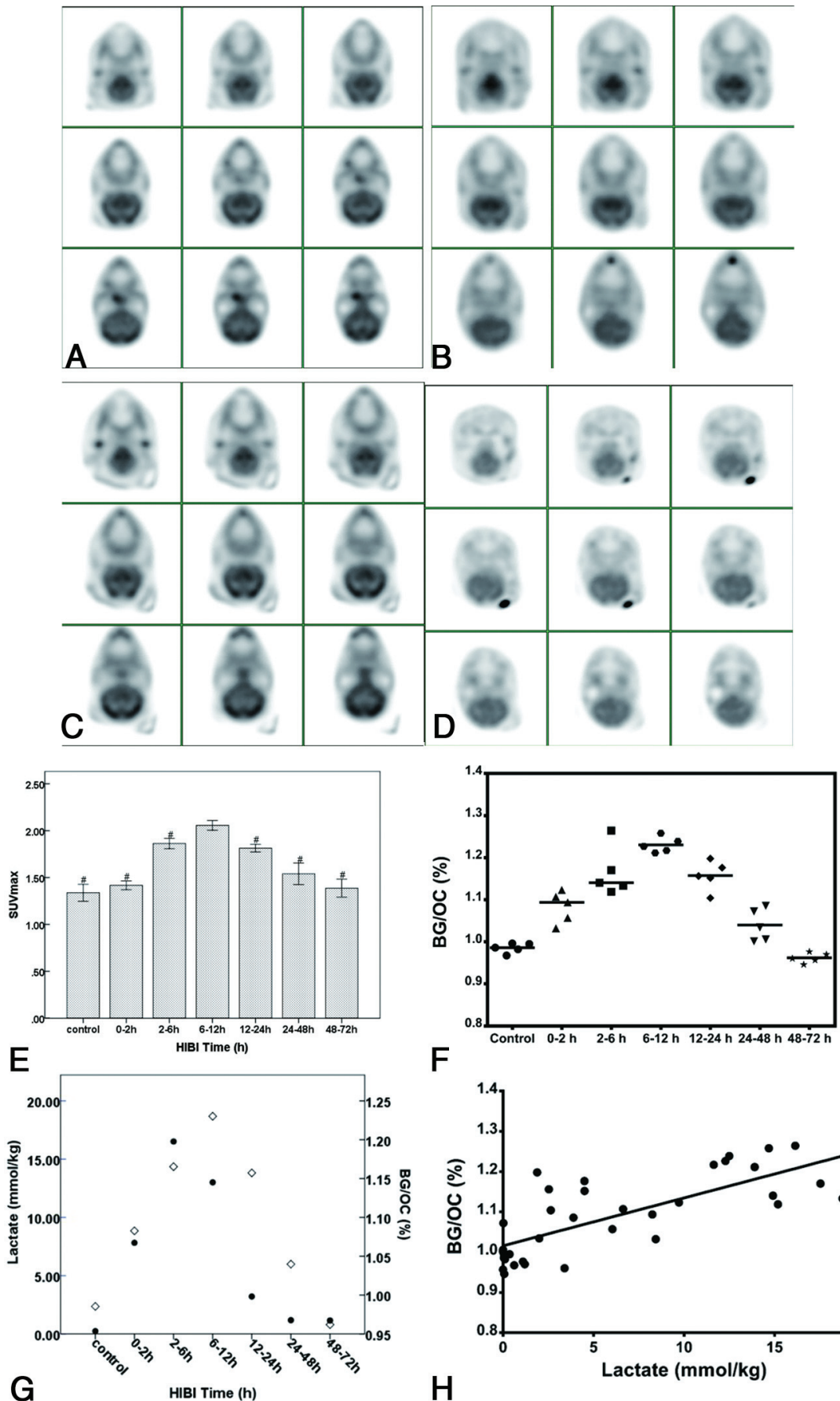

$\mathrm{F}$

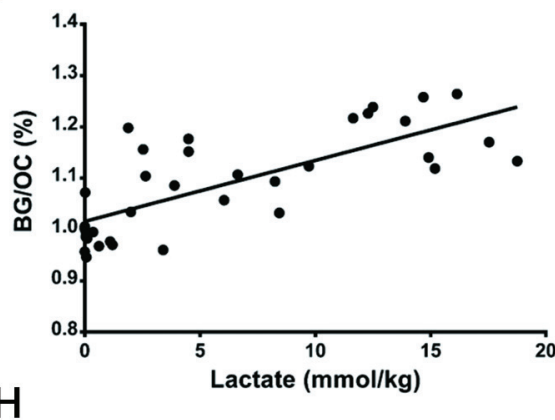

FIG 2. $\left[{ }^{18} \mathrm{~F}\right]$ FDG PET/CT images in the control group $(A)$ and the time points of $6-12,12-24$, and $48-72$ hours in the $\mathrm{HI}$ model groups $(B-D)$. Changes in the $S U V_{\max }$ of the basal ganglia and BG/OC ratio of the $B G$ in the control and $\mathrm{HI}$ model groups $(E-F)$. The $B G$ and $O C$ of the control group $(A)$ are clearly visible on $\left[{ }^{18} \mathrm{~F}\right] \mathrm{FDG}$ PET/CT images. $\left[{ }^{18} \mathrm{~F}\right] \mathrm{FDG}$ uptake was symmetrically distributed. At 6-12 hours after $\mathrm{HI}$, the $\mathrm{BG} / \mathrm{OC}$ ratio of the $\mathrm{HI}$ groups was greater than that of the control group (B). At 12-24 hours after $\mathrm{HI}$, the $\mathrm{BG} / \mathrm{OC}$ ratio in the $B \mathrm{G}$ was decreased compared with 6-12 hours (C). The BG/OC ratio was further decreased after 48-72 hours, and the BG and cortex showed less clearance $(D)$. The SUV ${ }_{\max }$ of the basal ganglia and BG/OC ratio peaked at 6-12 hours after HI. The hashtag represents a statistical difference compared with the 6- to 12-hour time points $(E-F)$. Changes in lactate levels and the BG/OC ratio after $\mathrm{HI}(G)$. The left vertical axis represents the lactate content, and the right vertical axis represents the $B G / O C$ ratio $(G$, the circle represents the average value of lactate content; the diamond represents the average value of BG/OC). Spearman correlation analysis showed that the lactate content was positively correlated with the BG/OC ratio $(r=0.74, P<.001 ; H)$. HIBI indicates hypoxic-ischemic brain injury. injection of the imaging agent. The PET slice thickness was $3.25 \mathrm{~mm}$. CT scanning parameters included the following: voltage, $80 \mathrm{kV}$; current, $50 \mathrm{~mA}$; slice thickness, $3.25 \mathrm{~mm}$. A 3D model was used in PET scanning.

\section{Image Processing}

PET/CT raw data were processed with Volume Viewer software on an AW4.6 workstation (GE Healthcare). The bilateral BG and occipital cortex (OC) on the same side were selected as the ROI. The size of the ROI of the BG was normalized to $1.0 \times 1.0 \mathrm{~cm}$, and that of the $\mathrm{OC}$, to $0.5 \times 0.5 \mathrm{~cm}$. The ROI was placed on the $\mathrm{BG}$ and the $\mathrm{OC}$ by reference to the CT images by 2 experienced radiologists. Maximum standard uptake values $\left(\mathrm{SUV}_{\text {max }}\right)$ of the BG/OC ratios were determined. The $\mathrm{SUV}_{\text {max }}$ of the BG and $\mathrm{BG} / \mathrm{OC}$ ratio reflects the uptake of $\left[{ }^{18} \mathrm{~F}\right]$ FDG in the BG.

\section{Histologic Examination and Immunohistochemical Analyses}

The fixed brain tissue was sliced in the coronal plane into 4-mm-thick sections. Slices containing the BG and hippocampus were used for immunohistochemical staining of MCT-2, MCT-4, glucose transporter-1 (GLUT-1), and GLUT-3 using antibodies purchased from Abcam (Bristol, England).

\section{Statistical Analyses}

All statistical analyses were performed with SPSS Software for Windows (Version 17.0; IBM, Armonk, New York). Data are presented as the mean \pm SD. Analysis of variance was used to analyze the data with equal variance followed by post hoc analysis with the least significant difference test. Spearman rank correlation analyses were conducted, and correlation coefficients and boxplot data were used. A $P$ value $<.05$ was considered statistically significant.

\section{RESULTS}

\section{Change in Lactate in the BG across Time after $\mathrm{HI}$}

${ }^{1} \mathrm{H}-\mathrm{MR}$ spectroscopy showed that after $\mathrm{HI}$, the lactate concentration increased first and then decreased but was still slightly higher than that of the control group at 48-72 hours (Fig 1 and Table). 

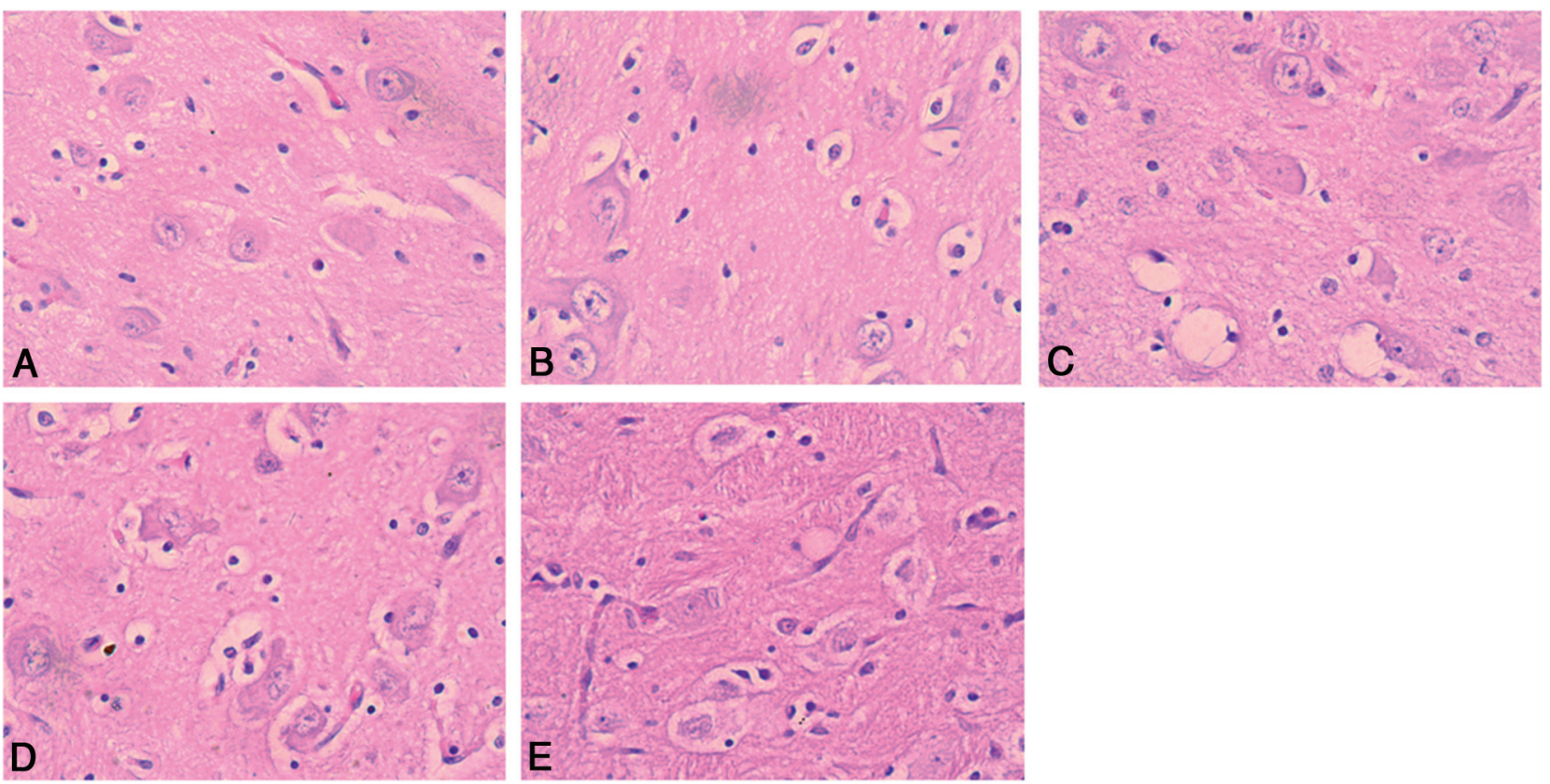

FIG 3. H\&E staining of the BG in the control and HI model groups. A-E, H\&E-stained images of the control group and the 6-, 24-, 48-, and 72-hour time points of the $\mathrm{HI}$ model groups. Compared with the control group $(A)$, we observed the following apparent pathologic changes: swelling in some astrocytes $(B)$, many astrocytes and some neurons apparently swollen with visible vacuoles and karyopyknosis $(C)$, and astrocytes apparently swollen and necrotic. Neurons also showed swelling and necrosis $(D)$, the neuronal membranes were damaged, and nuclei were swollen and weakly stained. These observations were made at $\times 400$ magnification $(E)$.

\section{PET/CT Results}

The radioactivity concentration was symmetrically distributed between the left and right hemispheres of the control group. The bilateral BG and OC were clearly visible and showed increased glucose metabolic activity, with radioactivity sparsely distributed in the white matter (Fig 2). There were significant differences in the $\mathrm{SUV}_{\max }$ of the BG and BG/OC between 6 and 12 hours and other time points $(P<.01)$. The $\mathrm{OC}$ and $\mathrm{BG}$ were still visible at 12-48 hours after HI. At 48-72 hours, the brain structures were less clear, and decreased metabolism was noted throughout the whole brain.

\section{Histologic Findings}

H\&E Staining and Expression Levels of MCT-2 and MCT-4 in the BG after HI. H\&E staining of the control group showed that the neurons were neatly arranged with intact cell morphology, abundant neuron plasma, and clear nucleoli. At 6 hours after HI, some glial cells were swollen with pale cytoplasm and intact neurons. At 24 hours after HI, a large portion of the glial cells was swollen with condensed nucleoli and the neurons were swollen and round with pale cytoplasm and bubbles. At 48 hours after HI, the glial cells were swollen and degraded, and the neurons were swollen with scattered visible neuronal necrosis. At 72 hours after HI, the neuronal cell membranes were broken and nuclei were swollen and pale. At different time points after $\mathrm{HI}$, the astrocytes and neurons had sustained different degrees of damage (Fig 3).

After HI, the expression of both MCT-2 and MCT-4 in the BG initially increased and then peaked at 12-24 hours (Fig 4). There were significant differences in MCT-2 and MCT-4 expression levels at 12-24 hours, compared with the control group and the other HI model groups $(P<.01)$.
Expression Levels of GLUT-1 and GLUT-3 in the BG after HI. The expression levels of GLUT-1 and GLUT-3 increased just after HI and peaked at 12-24 hours. The expression levels of both were significantly different at 12-24 hours after $\mathrm{HI}$ from the other time points $(P<.01)$. GLUT-1 expression decreased after 12-24 hours, as did GLUT-3 expression (Fig 5).

\section{DISCUSSION}

The results showed that lactate levels had peaked at 2-6 hours after $\mathrm{HI}$ and glucose peaked at 6-12 hours. The expression levels of the MCTs and GLUTs increased after HI (peak at 12-24 hours) and then decreased, and the study showed that astrocytes and neuronal damage after HI were not synchronized. Astrocytes damage occurred earlier and was more severe than neuronal damage; these findings were consistent with those of our previous study. ${ }^{10,13}$

It is likely more advantageous to detect lactate using ${ }^{1} \mathrm{H}-\mathrm{MR}$ spectroscopy at the $3 \mathrm{~T}$ field strength with a long TE of $288 \mathrm{~ms}$ because lipid T2 is short and the TE of the lipid signal declines; these features greatly distinguish lactate and lipid peaks. In $1.5 \mathrm{~T}$ field strength, a long TE of 144 ms usually shows an inverted lactate peak, which is therefore also easy to separate from the lipid peak. However, if a long TE (144 ms) is used with 3T, false-negative results may occur. ${ }^{14}$ In the present study, the use of a short TE ( $37 \mathrm{~ms}$ ) led to overlap of the lactate and lipid peaks, but use of LCModel separated the overlapped lactate and lipid signals and thus can still ensure the accuracy of the results.

In this study, the lactate content peaked at 2-6 hours and then gradually decreased. Several studies have shown that the increase in lactate concentration lasted for some time after HI. ${ }^{15}$ Pellerin et $\mathrm{al}^{16}$ proposed the ANLS hypothesis, which states that lactate, as a 

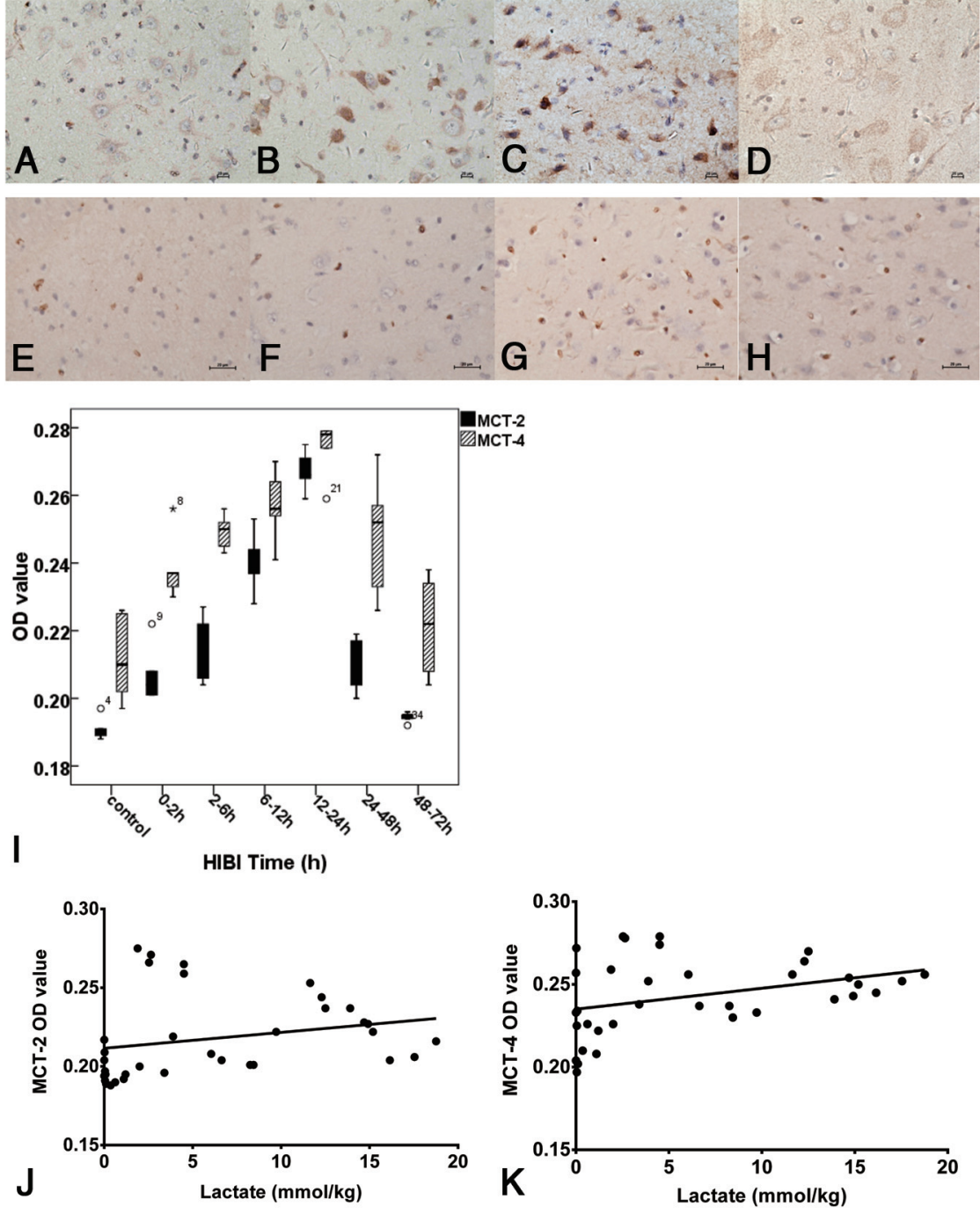

FIG 4. Expression levels of MCT- 2 and MCT-4 in the BG in the control and $H I$ model groups $(A-H)$ and change in expression levels of MCT-2 and MCT-4 in the BG after $\mathrm{HI}(I)$ and correlation with lactate content ( and $K$ ). A-D, MCT-2 expression in the control group and at 6,24 , and 48 hours after $\mathrm{HI}$ in the $\mathrm{HI}$ model groups. MCT-2 was mainly stained brown in the membrane of the neurons (A). Compared with the control group ( $A$ ), MCT-2 staining at 6 and 24 hours after $\mathrm{HI}$ ( $B$ and $C$ ) was darker and greater numbers of positive cells were observed. MCT-2 expression was reduced at 48 hours (D). E-H, MCT-4 expression in the BG of the control group and at 6, 24, and 48 hours after $\mathrm{HI}$ in the model groups. MCT-4 was mainly expressed in the membranes of astrocytes (E). MCT-4 staining was darker at 6 and 24 hours after $\mathrm{HI}(F$ and $G$ ), compared with the control group $(E)$. MCT-4 expression was reduced at 48 hours after $\mathrm{HI}(H)$. The expression levels of MCT-2 and MCT-4 were highest at 12-24 hours (I). Expression levels of MCT-2 and MCT-4 were positively correlated with the lactate level ( and K): MCT-2 $(r=0.47)$, MCT-4 $(r=0.38)$.

substrate of energy metabolism in neurons, was preferable to glucose as an energy source of neurons. ${ }^{17}$ In the current study, the regulation mechanism of lactate and glucose after HI was investigated on the basis of the ANLS hypothesis. A high concentration of lactate or hypoxia can induce activation of glycolytic enzymes $^{18,19}$ and further upregulate MCTs. Immunohistochemical analyses showed that after HI, the expression levels of MCT-2 and MCT-4 also increased, peaked around 12-24 hours, and then decreased. MCT-4 is primarily expressed in astrocytes and has a relatively low affinity for lactate, which facilitates the release of lactate, while MCT-2 is primarily expressed in neurons and has a high affinity for lactate; thus, characteristic kinetics are favorable for the uptake of lactate. ${ }^{20}$
Studies have shown that neurons can preferentially use lactate released from astrocytes. At normal physiologic concentrations of extracellular glucose and lactate, 79\% of the energy produced in neurons is from lactate and $21 \%$ is from glucose. Moreover, neurons can more rapidly use glucose and lactate than astrocytes, ${ }^{21,22}$ and histologic results also confirmed that neurons are damaged after astrocytes are damaged. ${ }^{10}$ Therefore, as an energy substrate of aerobic metabolism after HI, lactate has an important role in the protection of neurons after HI. ${ }^{23-25}$

After HI, the energy produced by lactate metabolism is not sufficient to maintain the normal physiologic activity of neurons. The increased expression levels of GLUT-1 and GLUT-3 and the increased use of glucose constitute a protective cellular response. The increase in lactate and glucose can temporarily compensate for the lack of energy in the brain in response to the needs of anaerobic solution. ${ }^{18,26}$

HI promotes abnormal glucose metabolism and is closely associated with brain cell necrosis. ${ }^{27} \mathrm{~A}$ decrease in the uptake of $\left[{ }^{18} \mathrm{~F}\right] \mathrm{FDG}$, to a certain degree, often implies irreversible brain injury. ${ }^{28}$ The results of the present study indicated that following HI, glucose metabolism increased and the BG/OC ratio peaked at around 6-12 hours. Glucose metabolism then decreased with time, and the uptake of $\left[{ }^{18} \mathrm{~F}\right]$ FDG in the whole brain decreased up to $48-72$ hours following HI. Lactate and glucose are critical for energy metabolism and interact closely with one another. After HI, glycolysis produces lactate; and because the energy generated from anaerobic metabolism is limited, the uptake and use of glucose by the brain are enhanced to supplement the energy shortage caused by HI. Therefore, an increase in lactate content is accompanied by an increase in glucose uptake.

Within 24 hours after HI, increased glucose uptake can temporarily supplement energy deficiencies, the glucose uptake is closely correlated with the upregulation of GLUTs at this stage. Immunohistochemical analyses showed that GLUT-1 and GLUT-3 synthesis increased after HI, peaked at 12-24 hours, and then decreased after 24 hours. Because GLUT-1 is mainly expressed in vascular endothelial cells, its increase helps transport glucose into the extracerebral space via the blood-brain barrier and increases glucose levels in the brain. ${ }^{29}$ GLUT- 3 is mainly ex- 

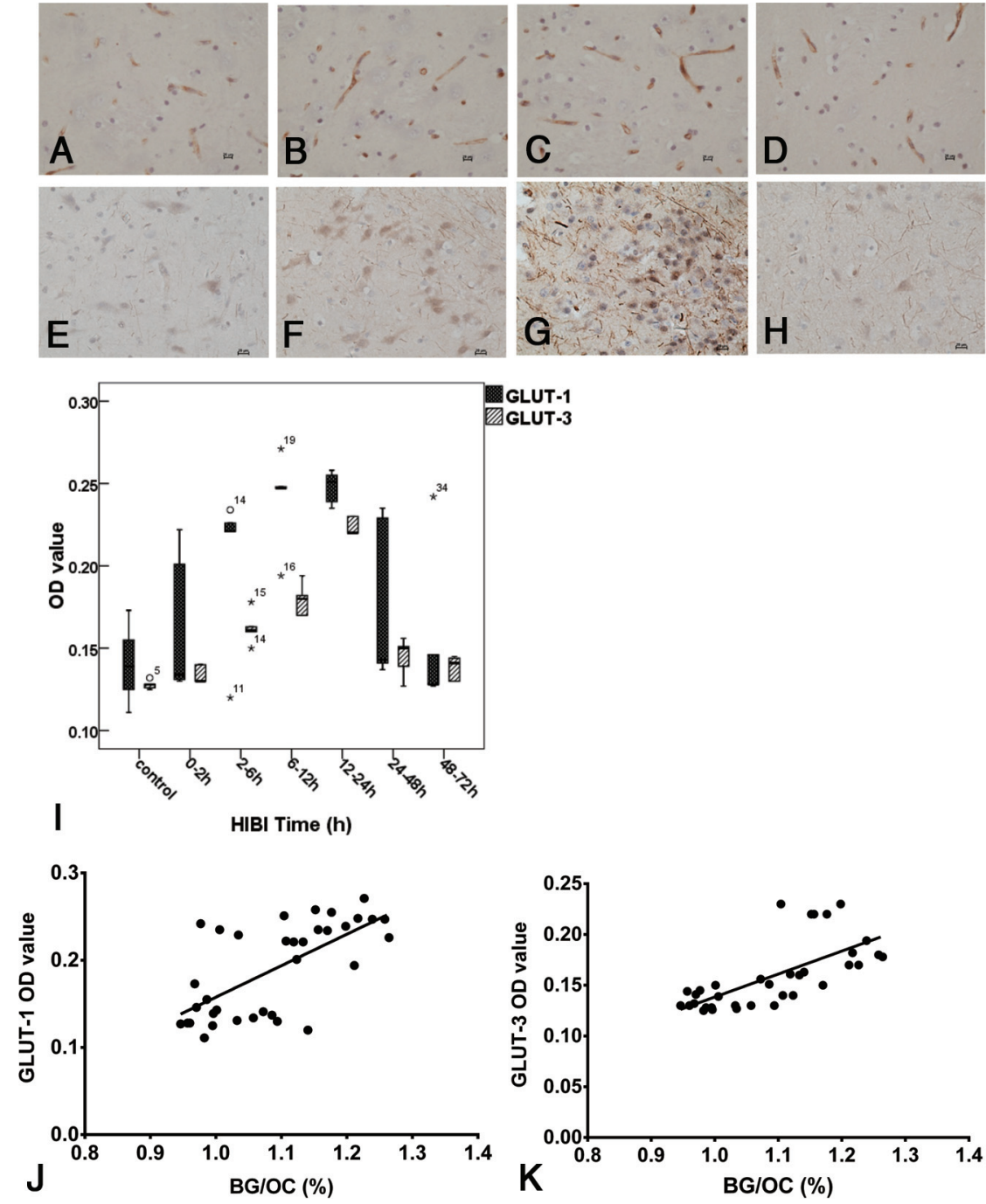

GLUT-1

GLUT-3

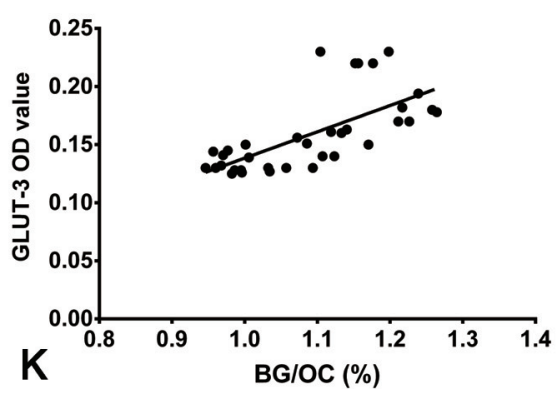

FIG 5. Expression levels of GLUT-1 and GLUT-3 in the BG in control and HI model groups $(A-H)$, immunohistochemical staining of GLUT-1 and GLUT-3 in the BG within 72 hours after HI (I), and correlation with BG/OC ( and K). A-D, GLUT-1 expression in the control group and at 6, 24, and 48 hours after $\mathrm{HI}$ in the $\mathrm{HI}$ model groups. GLUT-1 was mainly expressed in vascular endothelial cells (positive staining is shown as dark brown, rod-shaped structures) and astrocytes (A). Positively stained endothelial cells and astrocytes increased at 6 and 24 hours $(B$ and $C$ ) and decreased at 48 hours $(D)$ after HI. E-H, GLUT-3 expression in the control group and at 6, 24, and 48 hours after $\mathrm{HI}$ in the HI model groups. GLUT-3 was mainly expressed in neurons (E). The staining of the neuronal membrane was darker at 6 and 24 hours after $\mathrm{HI}(F$ and $G$ ). The staining lightened, and positively stained cells decreased at 48 hours after HI (H). GLUT-1 and GLUT-3 expression levels were highest at 12-24 hours (I). Spearman correlation analysis showed that the expression levels of GLUT-1 and GLUT-3 were positively correlated with the BG/OC ratio ( and K): GLUT-1 ( $r=0.64)$; GLUT-3 $(r=0.75)$.

pressed in neurons, and the upregulation of GLUT-3 promotes the transport of glucose from the extracerebral space into neurons. This increased glucose uptake improves energy metabolism. Upregulation of both GLUT-1 and GLUT-3 molecules temporarily supplements the energy deficiency and the demands of anaerobic glycolysis under HI conditions. ${ }^{18}$ At 48 hours after HI, astrocytes showed swelling and degeneration, while swelling and scattered necrosis of neurons was also observed. Up to 72 hours, many neurons were necrotic. Expression levels of GLUT-1 and GLUT-3 decreased after 24 hours. This decrease in GLUTs weakens the capacity to transport glucose, which reduces the uptake of $\left[{ }^{18} \mathrm{~F}\right]$ FDG.

Immediately after $\mathrm{HI}$, lactate production increased. The ANLS

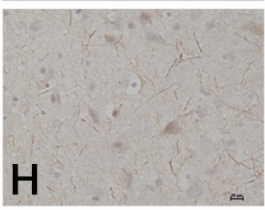

model can reflect lactate and hypoxia stimulation of MCTs and GLUTs. Up to the later stages of HI, necrosis of neurons and astrocytes disrupts coordination of the neuronal network and causes energy exhaustion. This series of changes in biochemical processes, including lactate, glucose, and associated transporters, coregulate energy metabolism after HI. ${ }^{30,31}$

The size, cardiovascular system, and brain maturity of neonate piglets and neonate children are highly similar. Therefore, the neonate piglet hypoxic-ischemic brain injury model and hypoxic ischemic encephalopathy are also similar. ${ }^{32}$ The success rate of modeling using piglets born within 24 hours is low with high mortality. Piglets at $3-5$ days of age can guarantee the success rate and meet the perinatal/neonatal brain requirements. Older neonate piglets do not meet the perinatal requirement but are more like older neonate children. Kyng et a ${ }^{33}$ used Danish Landrace piglets within 24 hours after birth, while neonate Yorkshire or Large White piglets 3-5 days of age were used in the present study. In this model, the bilateral internal carotid arteries were blocked and supplied with low oxygen concentrations. During the experiment, 2 piglets died, one due to excessive anesthesia and a second due to excessive blood loss. Nonetheless, this model is highly reproducible, with a high success rate and low mortality. Reperfusion was simulated during recovery of the blood supply, which is conducive to a reperfusion study. ${ }^{34-36}$ However, different anesthetics and anesthesia methods have different effects on neonatal brain injury. Using halothane to anesthetize animals may increase the degree of brain injury in the model. ${ }^{37}$ Propofol has a neuroprotective effect, which can reduce the degree of brain damage. ${ }^{38,39}$ In this study, Sumianxin was used as an anesthetic, and has the advantage of an effective anesthesia with smooth induction and recovery. The anesthesia time of Sumianxin is short and thus can reduce its impact on the model. ${ }^{19}$

\section{CONCLUSIONS}

These results suggest that lactate and glucose transporters have coordinated regulation of energy metabolism following HI.

\section{REFERENCES}

1. Itoh Y, Esaki T, Shimoji K, et al. Dichloroacetate effects on glucose and lactate oxidation by neurons and astroglia in vitro and on glu-

AJNR Am J Neuroradiol 39:569-76 Mar 2018 www.ajnr.org 
cose utilization by brain in vivo. Proc Natl Acad Sci U S A 2003;100: 4879-84 CrossRef Medline

2. Bouzat $P$, Oddo M. Lactate and the injured brain: friend or foe? Curr Opin Crit Care 2014;20:133-40 CrossRef Medline

3. Genc S, Kurnaz IA, Ozilgen M. Astrocyte-neuron lactate shuttle may boost more ATP supply to the neuron under hypoxic conditions-in silico study supported by in vitro expression data. BMC Syst Biol 2011;5:162 CrossRef Medline

4. Halestrap AP, Price NT. The proton-linked monocarboxylate transporter (MCT) family: structure, function and regulation. Biochem J 1999;343(Pt 2):281-99 Medline

5. Hertz L, Dienel GA. Lactate transport and transporters: general principles and functional roles in brain cells. J Neurosci Res 2005;79: 11-18 CrossRef Medline

6. Chiry O, Fishbein WN, Merezhinskaya N, et al. Distribution of the monocarboxylate transporter MCT2 in human cerebral cortex: an immunohistochemical study. Brain Res 2008;1226:61-69 CrossRef Medline

7. Amaral AI, Teixeira AP, Martens S, et al. Metabolic alterations induced by ischemia in primary cultures of astrocytes: merging $13 \mathrm{C}$ NMR spectroscopy and metabolic flux analysis. J Neurochem 2010; 113:735-48 CrossRef Medline

8. Pellerin L, Bouzier-Sore AK, Aubert A, et al. Activity-dependent regulation of energy metabolism by astrocytes: an update. Glia 2007; 55:1251-62 CrossRef Medline

9. Pellerin L, Magistretti PJ. How to balance the brain energy budget while spending glucose differently. J Physiology 2003;546(Pt 2):325 CrossRef Medline

10. Wang XY, Wang HW, Fu XH, et al. Expression of N-methyl-d-aspartate receptor 1 and its phosphorylated state in basal ganglia of a neonatal piglet hypoxic-ischemic brain injury model: a controlled study of (1)H MRS. Eur J Paediatr Neurol 2012;16:492-500 CrossRef Medline

11. Broad KD, Fierens I, Fleiss B, et al. Inhaled $45-50 \%$ argon augments hypothermic brain protection in a piglet model of perinatal asphyxia. Neurobiol Dis 2016;87:29-38 CrossRef Medline

12. Provencher SW. Automatic quantitation of localized in vivo $\mathbf{1 H}$ spectra with LCModel. NMR Biomed 2001;14:260-64 CrossRef Medline

13. Wang H, Wang X, Guo Q. The correlation between DTI parameters and levels of AQP-4 in the early phases of cerebral edema after hypoxic-ischemic/reperfusion injury in piglets. Pediatr Radiol 2012; 42:992-99 CrossRef Medline

14. Lange T, Dydak U, Roberts TP, et al. Pitfalls in lactate measurements at 3T. AJNR Am J Neuroradiol 2006;27:895-901 Medline

15. Thoren AE, Helps SC, Nilsson M, et al. The metabolism of C-glucose by neurons and astrocytes in brain subregions following focal cerebral ischemia in rats. J Neurochem 2006;97:968-78 CrossRef Medline

16. Pellerin L, Pellegri G, Bittar PG, et al. Evidence supporting the existence of an activity-dependent astrocyte-neuron lactate shuttle. Dev Neurosci 1998;20:291-99 CrossRef Medline

17. Cater HL, Chandratheva A, Benham CD, et al. Lactate and glucose as energy substrates during, and after, oxygen deprivation in rat hippocampal acute and cultured slices. J Neurochem 2003;87:1381-90 CrossRef Medline

18. Espinoza-Rojo M, Iturralde-Rodriguez KI, Chánez-Cárdenas ME, et al. Glucose transporters regulation on ischemic brain: possible role as therapeutic target. Cent Nerv Syst Agents Med Chem 2010;10: 317-25 CrossRef Medline

19. Zheng Y, Wang XM. Measurement of lactate content and amide proton transfer values in the basal ganglia of a neonatal piglet hypoxic-ischemic brain injury model using MRI. AJNR Am J Neuroradiol 2017;38:827-34 CrossRef Medline

20. Bergersen LH. Is lactate food for neurons? Comparison of monocarboxylate transporter subtypes in brain and muscle. Neuroscience 2007;145:11-19 CrossRef Medline
21. Gao C, Zhou L, Zhu W, et al. Monocarboxylate transporter-dependent mechanism confers resistance to oxygen- and glucose-deprivation injury in astrocyte-neuron co-cultures. Neurosci Lett 2015; 594:99-104 CrossRef Medline

22. Bouzier-Sore AK, Voisin $\mathrm{P}$, Canioni $\mathrm{P}$, et al. Lactate is a preferential oxidative energy substrate over glucose for neurons in culture. J Cereb Blood Flow Metab 2003;23:1298-306 CrossRef Medline

23. Moxon-Lester L, Sinclair K, Burke C, et al. Increased cerebral lactate during hypoxia may be neuroprotective in newborn piglets with intrauterine growth restriction. Brain Res 2007;1179:79-88 CrossRef Medline

24. Ogawa M, Watabe $\mathrm{H}$, Teramoto $\mathrm{N}$, et al. Understanding of cerebral energy metabolism by dynamic living brain slice imaging system with [18F]FDG. Neurosci Res 2005;52:357-61 CrossRef Medline

25. Berthet $\mathrm{C}$, Lei H, Thevenet J, et al. Neuroprotective role of lactate after cerebral ischemia. J Cereb Blood Flow Metab 2009;29:1780-89 CrossRef Medline

26. Royer C, Lachuer J, Crouzoulon G, et al. Effects of gestational hypoxia on mRNA levels of Glut 3 and Glut 4 transporters, hypoxia inducible factor-1 and thyroid hormone receptors in developing rat brain. Brain Res 2000;856:119-28 CrossRef Medline

27. Hardy OT, Hernandez-Pampaloni M, Saffer JR, et al. Diagnosis and localization of focal congenital hyperinsulinism by $18 \mathrm{~F}$-fluorodopa PET scan. J Pediatr 2007;150:140-45 CrossRef Medline

28. Thorngren-Jerneck K, Ley D, Hellstrom-Westas L, et al. Reduced postnatal cerebral glucose metabolism measured by PET after asphyxia in near term fetal lambs. J Neurosci Res 2001;66:844-50 CrossRef Medline

29. Vemula S, Roder KE, Yang T, et al. A functional role for sodiumdependent glucose transport across the blood-brain barrier during oxygen glucose deprivation. J Pharmacol Exp Ther 2009;328:487-95 CrossRef Medline

30. Brown AM, Ransom BR. Astrocyte glycogen and brain energy metabolism. Glia 2007;55:1263-71 CrossRef Medline

31. Mehta SL, Manhas N, Raghubir R. Molecular targets in cerebral ischemia for developing novel therapeutics. Brain Res Rev 2007;54: 34-66 CrossRef Medline

32. Buckley NM. Maturation of circulatory system in three mammalian models of human development. Comp Biochem Physiol A Comp Physiol 1986;83:1-7 Medline

33. Kyng KJ, Skajaa T, Kerrn-Jespersen S, et al. A piglet model of neonatal hypoxic-ischemic encephalopathy. J Vis Exp 2015:e52454 CrossRef Medline

34. Roohey T, Raju TN, Moustogiannis AN. Animal models for the study of perinatal hypoxic-ischemic encephalopathy: a critical analysis. Early Hum Dev 1997;47:115-46 CrossRef Medline

35. Zhang YF, Wang XY, Guo F, et al. Simultaneously changes in striatum dopaminergic and glutamatergic parameters following hypoxic-ischemic neuronal injury in newborn piglets. Eur J Paediatr Neurol 2012;16:271-78 CrossRef Medline

36. Zhang YF, Wang XY, Cao L, et al. Effects of hypoxic-ischemic brain injury on striatal dopamine transporter in newborn piglets: evaluation of 11C-CFT PET/CT for DAT quantification. Nucl Med Biol 2011;38:1205-12 CrossRef Medline

37. Thoresen M, Simmonds M, Satas S, et al. Effective selective head cooling during posthypoxic hypothermia in newborn piglets. Pediatr Res 2001;49:594-99 CrossRef Medline

38. Lingwood BE, Dunster KR, Healy GN, et al. Cerebral impedance and neurological outcome following a mild or severe hypoxic/ischemic episode in neonatal piglets. Brain Res 2003;969:160-67 CrossRef Medline

39. Björkman ST, Foster KA, O’Driscoll SM, et al. Hypoxic/ischemic models in newborn piglet: comparison of constant FiO2 versus variable FiO2 delivery. Brain Res 2006;1100:110-17 CrossRef Medline 